DOI:

ARTICULO ORIGINAL

\title{
Epidemiológica del ataque cerebro vascular en un hospital universitario
}

Javier Gaudiano ORCID: 0000-0003-3824-4695 Asistente Clínica Médica. UdelaR.

Diego Graña ORCID: 0000-0001 8979-4692 Profesor Adjunto Clínica Médica. UdelaR.

Mabel Goñi ORCID: 0000-0001-8593-6569 Profesor Director Clínica Médica. UdelaR.

Virginia Colina* ORCID: 0000-0002-3559-0800

Andrea Cosentino* ORCID: 0000-0001-7885-512X

Romina Pensado* ORCID: 0000-0002-6637-9717

Victoria Ruglio* ORCID: 0000-0003-3653-367X

Magali Scaron* ORCID: 0000-0002-4679-0250

Leticia Vidart* ORCID: 0000-0002-3874-1600

*Bachiller de Medicina (5to año). Facultad de Medicina. UdelaR.

\author{
Epidemiological of cerebrovascular attack in a university hospital \\ Epidemiologia do ataque cerebrovascular em hospital universitário
}

Resumen. Introducción: El ataque cerebro vascular es una enfermedad prevalente en nuestro medio con elevada morbimortalidad. El Hospital Pasteur es un centro de tercer nivel, que asiste un elevado número de pacientes con esta patología. Conocer los datos epidemiológicos de esta afección permitirá desarrollar medidas de promoción de salud y prevención primaria. Identificar la forma de presentación clínica y los algoritmos de estudio, permitirán un adecuado diagnóstico, tratamiento precoz y desarrollo de medidas de prevención secundaria. Objetivos: Conocer las características sociodemográficas de la población con diagnóstico de ataque cerebro vascular o accidente isquémico transitorio asistidas en las salas de medicina del Hospital Pasteur, los factores de riesgo asociados, las formas de presentación clínica y precisar si se cumplió con el algoritmo diagnóstico propuesto. Métodos y procedimiento: Estudio descriptivo observacional y transversal realizado en el Hospital Pasteur. La población de estudio fueron pacientes adultos ingresados en sala de medicina con diagnóstico de ataque cerebro vascular o accidente isquémico transitorio que presentaron el evento durante su internación, en el periodo comprendido entre Julio y Setiembre de 2018. Resultados y discusión: Se recabaron datos de 29 pacientes, 20 de sexo femenino. La media de edad fue de 70.34 años. La naturaleza isquémica fue la más prevalente. Los factores de riesgo cardiovascular más frecuentes fueron HTA, sedentarismo, dislipemia y tabaquismo. La principal forma de presentación fue síndrome piramidal. Se cumplió con el algoritmo diagnostico en todos los pacientes. Conclusión: Conocer los factores de riesgo, naturaleza y forma de presentación clínica permite elaborar estrategias de prevención primaria y secundaria para el abordaje integral de estos pacientes, intentando así reducir la incidencia y secuelas de esta enfermedad.

Palabras clave: ACV, AIT, factores de riesgo cardiovascular, HTA.

Abstract. Introduction: Acute ischemic stroke is a prevalent condition in our working environment, with high morbility and mortality. Hospital Pasteur is a tertiary level institution, which assists an elevated number of patients with acute ischemic stroke. Getting to know its epidemiologic characteristics will enable the development of health promotion and primary prevention measures. Identifying its clinical presentation form and applying validated study algorithms will allow for a proper diagnosis, early treatment and development of secondary prevention measures. Objectives: To study the sociodemographic characteristics of patients diagnosed with acute ischemic stroke or transient ischemic attack assisted in Hospital Pasteur's Internal Medicine wards, their associated risk factors, clinical presentation and to determine whether the proposed study algorithm was followed. Methodology and procedure: This is a descriptive, observational and transversal study which took place at Hospital Pasteur. The population consisted of adult patients admitted to Internal Medicine wards with the diagnosis of acute ischemic stroke, transient ischemic attack or who presented the event during their stay, between the months of July and September 2018. Results and discussion: The data of 29 patientes was obtained; 20 were female. The mean age was 70.34 years. Ischemic nature was the most prevalent. The most frequent risk factors were arterial hypertension, sedentary lifestyle, dyslipidemia and smoking. Pyramidal syndrome was the most common clinical presentation. A study algorithm was followed in all patients. Conclusion: Getting to know the risk factors, nature and clinical presentation form of AIS and TIA allows for the creation of primary and secondary prevention strategies for the comprehensive approach of these patients, therefore intending to reduce this disease's incidence and its terrible consequences. 
Keywords: AIS, TIA, cardiovascular risk factors, arterial hypertension.

Resumo. Introdução: O ataque cerebrovascular é uma doença prevalente em nosso meio, com alta morbimortalidade. O Hospital Pasteur é um centro de terceiro nível, que auxilia um grande número de pacientes com essa patologia. Conhecer os dados epidemiológicos dessa condição permitirá o desenvolvimento de ações de promoção da saúde e prevenção primária. Identificar a forma de apresentação clínica e os algoritmos do estudo permitirá um diagnóstico adequado, tratamento precoce e desenvolvimento de medidas de prevenção secundária. Objetivos: Conhecer as características sociodemográficas da população com diagnóstico de ataque cerebrovascular ou ataque isquêmico transitório assistido nas salas médicas do Hospital Pasteur, os fatores de risco associados, as formas de apresentação clínica e determinar se o algoritmo de diagnóstico proposto foi atendido. Métodos e procedimentos: Estudo observacional e transversal descritivo realizado no Hospital Pasteur. A população do estudo foi de pacientes adultos admitidos na enfermaria com diagnóstico de ataque cerebrovascular, ataque isquêmico transitório que apresentaram o evento durante sua internação, no período entre julho e setembro de 2018. Resultados e discussão: Os dados foram coletados de 29 pacientes, sendo 20 do sexo feminino. A idade média foi de 70,34 anos, sendo a natureza isquêmica a mais prevalente. Os fatores de risco cardiovascular mais frequentes foram hipertensão arterial, sedentarismo, dislipidemia e tabagismo. A principal forma de apresentação foi a síndrome piramidal. $O$ algoritmo de diagnóstico foi preenchido em todos os pacientes. Conclusão: Conhecer os fatores de risco, natureza e forma de apresentação clínica permite desenvolver estratégias de prevenção primária e secundária para abordagem abrangente desses pacientes, buscando reduzir a incidência e sequelas da doença.

Palavras-chave: ACV, AIT, fatores de risco cardiovascular, HTA

Recibido: 20/02/2019 Aceptado: 16/05/2019

Clínica Médica "2". Hospital Pasteur. Facultad de Medicina. Universidad de la República (UdelaR). Asistencia Servicios Salud del Estado (ASSE). Montevideo, Uruguay. 


\section{Introducción}

El ataque cerebro vascular (ACV) es una enfermedad con alta prevalencia en nuestro medio, la misma es de 8,6 casos por cada 1.000 habitantes, y posee una incidencia de 181,3 casos cada 100.000 habitantes ${ }^{(1)}$. Presenta una elevada morbimortalidad con secuelas de impacto negativo en la calidad de vida de los pacientes, con altos costos sanitarios y sociales. En nuestro país constituye la segunda causa de muerte luego del infarto agudo de miocardio ${ }^{(2)}$.

Se han realizado diversos estudios con distintos enfoques sobre esta patología, pero ninguno orientado a la epidemiología. En $2004{ }^{(3)}$ se recaban datos del Hospital Pasteur en esta patología.

El objetivo general del trabajo fue determinar las características de la población con diagnóstico de ACV o accidente isquémico transitorio (AIT) asistidas en las salas de medicina del Hospital Pasteur. Como objetivos específicos fueron describir las características sociodemográficas, los factores de riesgo asociados, las formas de presentación clínica de los pacientes con ACV o AIT y precisar si se cumplió con el algoritmo diagnóstico propuesto.

Conocer los datos epidemiológicos de esta afección permitirá desarrollar medidas de promoción de salud y prevención primaria haciendo hincapié en cambios en el estilo de vida y tratamiento de los factores de riesgo. Identificar la forma de presentación clínica permitirá un adecuado diagnóstico, tratamiento precoz en agudo y medidas de prevención secundaria en la evolución.

\section{Material y Métodos}

Se trata de un estudio descriptivo, observacional, de tipo transversal, que incluyó los pacientes adultos ingresados en salas de medicina del Hospital Pasteur (Montevideo, Uruguay) con diagnóstico de ACV, AIT o que instalaron el cuadro durante la internación, en el periodo comprendido entre el 10 de Julio y el 15 de Setiembre de 2018. El tamaño de la muestra está relacionado con la cantidad de casos que se presentaron durante dicho período de tiempo. Se excluyeron los pacientes menores de 18 años y aquellos que se negaron a participar.

La recolección de datos se obtuvo mediante revisión de historias clínicas seleccionando información a través de un formulario preestablecido. Las variables a observar fueron: edad, sexo, raza, hipertensión arterial, diabetes, dislipemia, obesidad, sedentarismo, hiperuricemia, tabaquismo, alcoholismo, trombofilias, cardiopatía de base, consumo de anticoagulantes, ACV previo, AIT previo, enfermedad renal crónica, forma de presentación clínica. inicial.

En la figura 1 se presenta el algoritmo de estudios para la valoración diagnóstica y etiológica

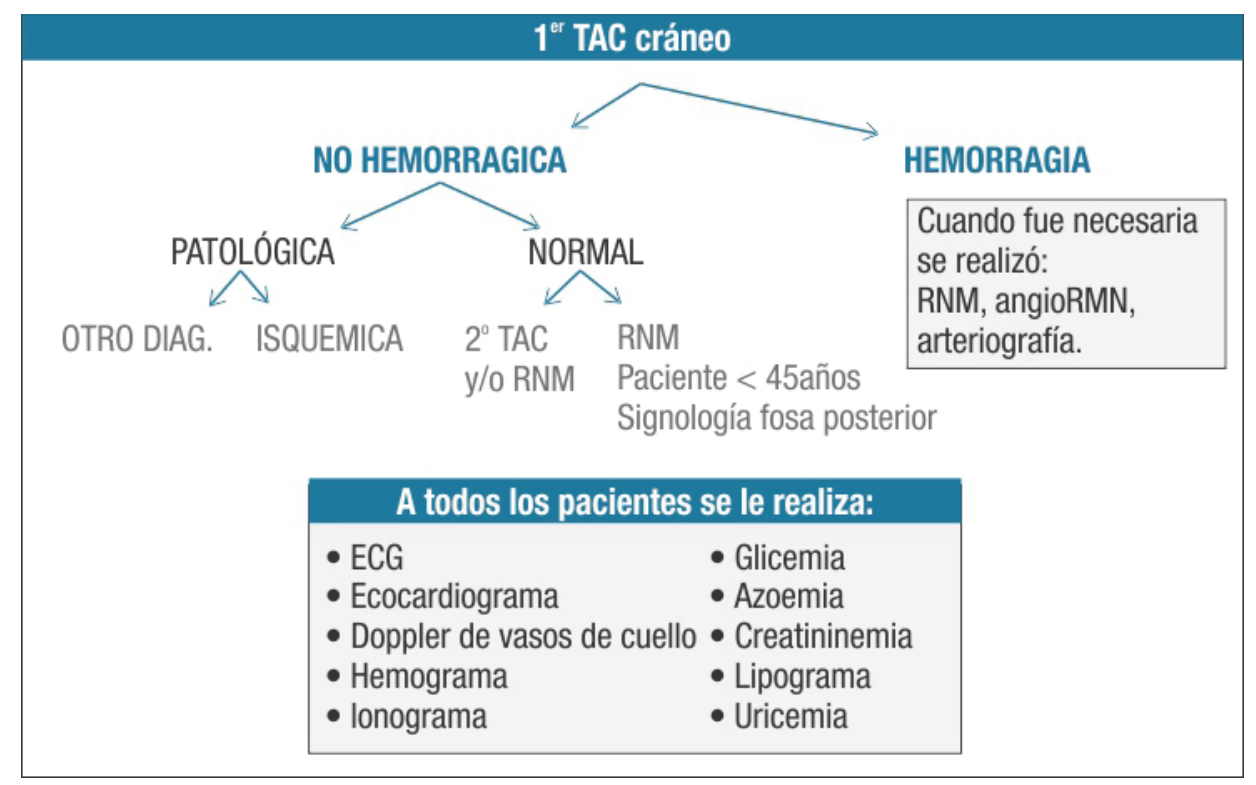


Las variables utilizadas son categóricas representadas en números absolutos. Para el análisis de los datos se utilizó el archivo de base de datos Epilnfotm versión 7.2.2.6. y para la elaboración de los gráficos se utilizó Excel 2010.

Los datos recolectados fueron utilizados únicamente con fines académicos. La participación del estudio fue de forma voluntaria y anónima. Todos los participantes del mismo recibieron información clara y comprensible sobre la justificación y metodología de trabajo. El proyecto de investigación fue aprobado por el comité de ética de la Facultad de Medicina el día 10 de julio de 2018.

\section{Resultados}

En el período analizado 31 pacientes cumplieron con los criterios diagnósticos, de los cuáles 1 se negó a participar del estudio, y otro paciente no fue capaz de brindar su consentimiento; por lo que 29 pacientes conforman la totalidad que se analizó.

De ellos, 28 fueron ACV de tipo isquémico y 1 de tipo hemorrágico.

Todos los integrantes fueron de raza blanca.

Veinte ocurrieron en pacientes de sexo femenino y nueve de sexo masculino La media de edad fue de $70.34 \pm 9.7$ años. La media de edad para las mujeres fue de 71,90 $\pm 10,44$ años y para los hombres fue de $67,00 \pm 7,58$ años. El mínimo de edad fue de 53 años y el máximo de 89. El rango etario donde se presentó la mayor cantidad de ACV fue entre 70-80 años con 13 pacientes, de los cuales 9 fueron mujeres y 4 hombres.

Respecto a los factores de riesgo, en orden de prevalencia, los resultados fueron: 24 pacientes con HTA, 19 con sedentarismo, 14 con dislipemia, 12 pacientes tabaquistas, 12 con cardiopatía de base ( 6 isquémicas, 4 valvulares, 2 hipertensiva), 9 presentaron ACV previo, 7 con DM, 6 con obesidad, 6 con arritmia (4 fibrilación auricular, 1 bloqueo auriculo ventricular Mobitz II, 1 Wolff Parkinson White), 5 pacientes alcohólicos, 5 pacientes anticoagulados (1 por cardiopatía valvular y 4 por fibrilación auricular), 4 con hiperuricemia, 2 presentaron AIT previo y 1 con enfermedad renal crónica. Figura 2.

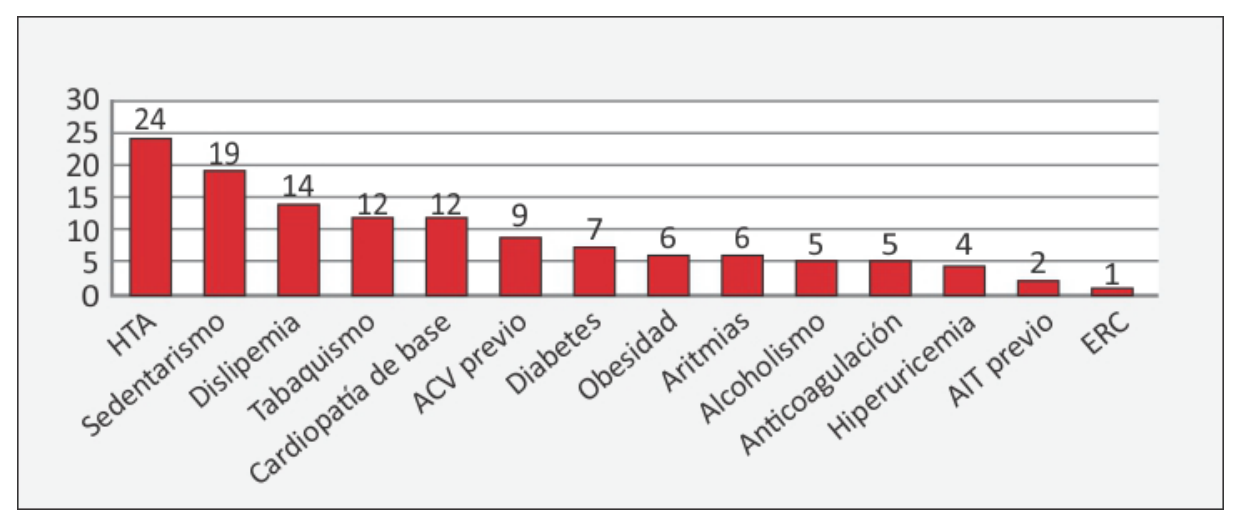

En la tabla 1 se presenta la asociación de los factores de riesgo encontrados. 20 pacientes asociaron tres o más, 4 pacientes asociaron dos factores de riesgo, 4 pacientes se presentaron con un único factor de riesgo y 1 paciente no presentó ninguno.

\begin{tabular}{|l|l|}
\multicolumn{1}{|c|}{ Numero de Factores de riesgo Frecuencia (n=29) } \\
\hline 0 & 1 \\
\hline 1 & 4 \\
\hline 2 & 4 \\
\hline$\geq 3$ & 20 \\
\hline
\end{tabular}

De la clínica descrita al ingreso, 24 pacientes se presentaron con síndrome piramidal, 19 con síndrome simbólico, 6 pacientes con síndrome sensitivo, 3 con síndrome sensorial visual, 6 con síndrome de fosa posterior, 1 paciente con síndrome de hipertensión endocraneana y 1 con síndrome frontal. Ningún paciente presentó síndrome meníngeo. 22 pacientes asociaron más de 
1 síndrome en su presentación clínica. La asociación con mayor incidencia fue la del síndrome piramidal con el síndrome simbólico observado en 17 pacientes. Figura 3.

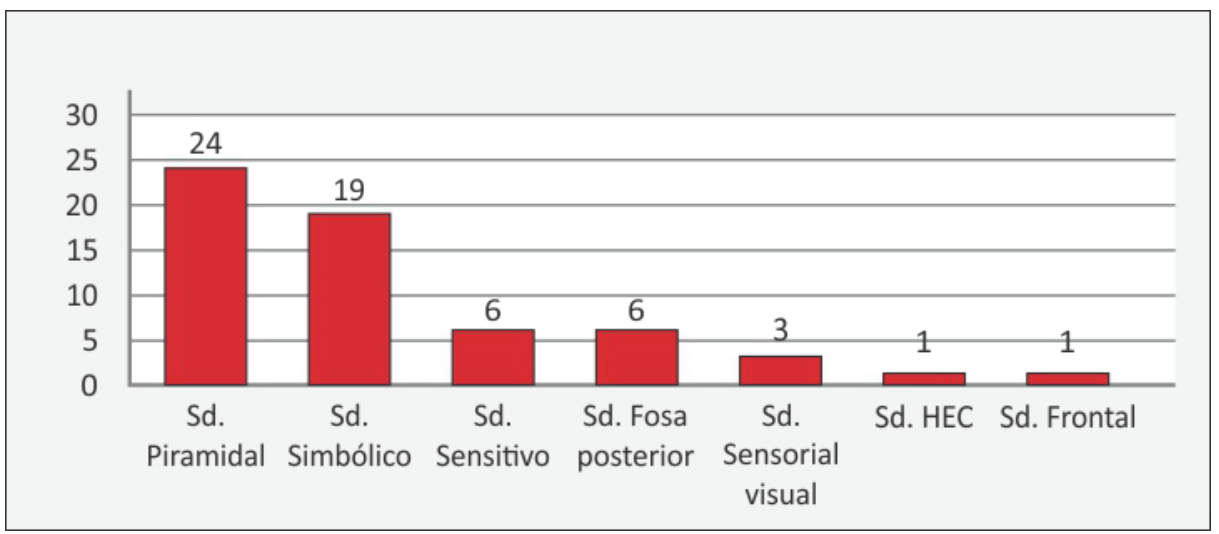

En cuanto a la paraclínica, se le realizó tomografía computada (TAC) cráneo a la totalidad de los pacientes, resonancia nuclear magnética (RNM) cráneo a 3 y angioTAC a 2 pacientes (según algoritmo), doppler de vasos de cuello a 28 pacientes, ECG y ecocardiograma a 26 . Función renal, ionograma y hemograma a 28 pacientes, y perfil lipídico a 17.

Destacamos que esta investigación se realizó sobre una población acotada por incluir solamente pacientes internados en salas de medicina, excluyendo pacientes que consultaron en emergencia y fueron derivados a centros especializados, ingresados a cuidados intensivos, o que fallecieron al ingreso. Así como el breve período de tiempo para recabar los datos.

\section{Discusión}

La totalidad de pacientes recabados en nuestro estudio superaron los 50 años. El rango etario donde se documentó el mayor número de casos fue el de 70-80 años. Varios estudios epidemiológicos establecen que, a mayor edad, mayor es el riesgo de padecer un evento vascular encefálico, coincidiendo con los hallazgos en nuestro trabajo. ${ }^{(4,5)}$

Como era de esperar, la naturaleza isquémica se presentó en la mayoría de nuestros pacientes (28/29 pacientes). A su vez, nuestra población predominantemente de sexo femenino $(n=20)$. Según la literatura no se han encontrado diferencias en la incidencia de ACV respecto al sexo, aunque ésta es superior en mujeres a medida que aumenta la edad. ${ }^{(4,6)}$.Coincidiendo con nuestra investigación donde el rango etario de mayor prevalencia presentó 9 mujeres y 4 hombres.

Respecto a los factores de riesgo cardiovasculares, el más prevalente fue la HTA, resultado acorde con otros estudios nacionales donde también predomina como principal factor (7), vinculando el aumento de cifras de presión arterial hasta dos tercios de los ACV ${ }^{(8,9)}$. Junto con la edad, constituyen los factores de riesgo más significativos. ${ }^{(10,11)}$

El sedentarismo fue el segundo factor de riesgo cardiovascular más frecuente en nuestro estudio comprometiendo 19 pacientes. Según la OMS, 60\% de la población mundial no realiza actividad física. ${ }^{(12,13)}$ Diversos artículos internacionales, analizaron que quienes realizan actividad física en forma moderada tienen un menor riesgo de padecer enfermedades cardiovasculares en relación a las personas inactivas. ${ }^{(14)}$

La dislipemia presentó una frecuencia de 14 pacientes, en acuerdo la relación documentada que existe entre este factor de riesgo y patología cerebrovascular. ${ }^{(15)}$

Si bien en trabajos internacionales se comprobó que la tasa de incidencia de ACV isquémico es de 3 a 4 veces mayor para pacientes con DM que en pacientes no diabéticos. ${ }^{(16)}$ Esta asociación no se presentó en nuestro estudio, registrándose DM sólo en 7 pacientes.

En cuanto a los antecedentes de AIT y ACV previo, está descrito que éstos constituyen un factor de riesgo para padecer un nuevo evento. ${ }^{(17,18)}$ Esto es concordante con lo hallado en nuestro estudio, donde se observó que 11 de 29 pacientes habían padecido un evento vascular previamente, correspondiendo esto a más de un tercio del total. 
De los 29 pacientes, 5 recibían anticoagulantes al momento de presentar el evento. No contamos con datos del rango terapéutico al momento de instalar el episodio.

Nuestros datos coinciden con la revisión según Fuentes y cols. donde 1 de cada 6 ictus ocurren en pacientes con FA. ${ }^{(12)}$ En nuestra muestra 4 pacientes presentaron fibrilación auricular al inicio de los síntomas.

El tabaquismo fue uno de los factores de riesgo más relevantes observados en nuestro estudio, donde 12 del total de los ACV presentaron este antecedente. De acuerdo a lo visto en las guías, el consumo de tabaco aumenta los riesgos de padecer esta patología y el abandono del hábito disminuye el riesgo de sufrir enfermedades vasculares. ${ }^{(12,19)}$

A pesar de que el consumo de alcohol, ERC, hiperuricemia y las trombofilias están documentados como factores de riesgo para ACV, ${ }^{(12,20,21)}$ no fueron prevalentes en nuestra población estudiada.

En lo que respecta a la presentación clínica, en nuestra muestra el síndrome piramidal fue el más prevalente, seguido del síndrome simbólico, evidenciando la arteria cerebral media como la más afectada. ${ }^{21,22)}$ El resto de los síndromes no mostraron una incidencia significativa. Estos datos clínicos concuerdan con estudios nacionales, en donde la afectación motora es uno de los principales motivos de consulta en el contexto de ACV. ${ }^{(23,24)}$ En 22 pacientes se presentaron más de un síndrome al momento del diagnóstico, 17 de los mismos se presentaron con síndrome piramidal y elementos de síndrome simbólico asociado, siendo esta asociación la principal forma de presentación en nuestro hospital.

En cuanto al algoritmo diagnostico descrito anteriormente, podemos concluir que el mismo se cumplió en todos los pacientes analizados. Confirmamos que en todos los casos se realizó una TC de cráneo, sabemos que su gran ventaja es la de establecer con gran especificidad el diagnóstico diferencial entre el ictus isquémico y el hemorrágico. ${ }^{(25)}$ En los casos con signología de fosa posterior se realizó RMN (3 casos) y en los que según el algoritmo correspondía se realizó angioTAC, útil para detectar malformaciones vasculares cerebrales.

Si bien se cumplió con el algoritmo diagnóstico, notamos que el análisis de perfil lipídico sólo fue realizado en 17 de los 29 pacientes. El mismo cobra importancia cuando se realiza en las primeras 24 horas de instalados los síntomas, pero debería realizarse en todos los pacientes por ser rápido y accesible.

\section{Conclusiones}

El ACV prevaleció en la población de sexo femenino, raza blanca y más envejecida.

La naturaleza isquémica fue la más frecuente y la totalidad de los pacientes presentaron FRCV confirmando el estrecho vínculo entre ellos y el ACV. La HTA y sedentarismo fueron los más prevalentes. La presentación clínica más frecuente fue el síndrome piramidal asociado al compromiso simbólico. En todos los pacientes estudiados se cumplió con el algoritmo diagnóstico para la búsqueda de la etiología.

Conocer los factores de riesgo, naturaleza y forma de presentación clínica permite elaborar estrategias de prevención primaria y secundaria para el abordaje integral de estos pacientes, intentando así reducir la incidencia y secuelas de esta enfermedad. 


\section{Bibliografía}

1- 1. Hochman B, Coelho J, Segura J, Galli M, Ketzoian C, Pebet M. Incidencia del accidente cerebrovascular en la ciudad de Rivera, Uruguay. Rev Neurol. 2006; 43: 78-83.

2- 2. Uruguay. Comisión Honoraria para la Salud Cardiovascular. Área de Epidemiología y Estadística. Mortalidad por enfermedades cardiovasculares en el Uruguay 2008. Montevideo: CHSC, 2010

3- 3. Dufrechou C, Perendones M, Cedrés S, Grille S, Muyano A, Tenaglia K, et al. Prevalencia de "Stroke" en un hospital general de adultos. XXXIV Congreso Nacional de Medicina Interna -Sesión Temas libres; 2005 Nov. 29 - 2 Dic; IMM, Montevideo. Montevideo: Colegio de Enfermeras del Uruguay Sociedad de Medicina Interna del Uruguay; 2005.

4- 4. Martin M, Poveda J, Segura M, Portela V, Coelho G. Factores de riesgo de las enfermedades cerebrovasculares en Pedras, Maranhão, Brasil [Internet]. Rev. Inf. Cient. 2018; 90:29-37[cited 6 October 2018]. Available from: http://www.revinfcientifica.sld.cu/index.php/ric/article/view/1815/3526.

5- 5. Berenguer Guarnaluses LJ, Pérez Ramos A. Factores de riesgo de los accidentes cerebrovasculares durante un bienio. MEDISAN. 2016 Mayo; 20( 5 ): 621-629.

6- 6. Camejo C, Legnani C, Gaye A, Arcieri B, Brumett F, Castro L, et al . Unidad de ACV en el Hospital de Clínicas: comportamiento clínico-epidemiológico de los pacientes con ACV (2007-2012). Arch. Med Int. 2015 Mar; 37( 1 ): 30-35

7- 7. Arias-Rivas S, Vivancos-Mora J, Castillo J. Epidemiología de los subtipos de ictus en pacientes hospitalizados atendidos por neurólogos: Resultados del registro EPICES (I) [Internet]. Rev Neurol. 2012; 54(7): 385-393 [cited 6 October 2018]. Available from: https://doi.org/10.33588/rn.5407.2011551

8- 8. Lawes CM, Vander Hoorn S, Law MR, Elliott P, MacMahon S, Rodgers A. Blood pressure and the global burden of disease 2000. Part II: estimates of attributable burden. J Hypertens. 2006 Mar;24(3):423-30.

9- 9. Organización Panamericana de la Salud. La OPS/OMS insta a las personas en las Américas a chequear su presión arterial para prevenir infartos y accidentes cerebrovasculares. [online] OPS: Montevideo, 2014 [Accessed 1 Oct. 2018]. Available at: https://www.paho.org/uru/index. php?option=com_content\&view=article\&id=844:la-opsoms-insta-a-las-personas-en-las-americas-achequear-su-presion-arterial-para-prevenir-infartos-y-accidentes-cerebrovasculares\&ltemid=340.

10- 10. Castilla-Guerra L, Fernández-Moreno MC, Romera-Tellado M, Álvarez-Suero J. Prevención primaria del ictus en ancianos: evidencia actual en el tratamiento de la hipertensión arterial. Rev. esp. geriatr. gerontol. 2012; 47(3):119-124.

11- 11. Peral MA, Fracchi L, Chahla R. Hipertensión arterial como factor de riesgo mayor en la prevalencia de accidente cerebrovascular e infarto agudo de miocardio en la provincia de Tucumán. Rev. invest. salud Ministerio de Salud Pública de Tucumán. 2017; 1(1): 3-7.

12- 12. Fuentes B, Gállego J, Gil-Nuñez A, Morales A, Purroy F, Roquer J, et al. Guía para el Tratamiento Preventivo del Ictus Isquémico y AIT (I). Actuación sobre los factores de riesgo y estilo de vida. Neurología 2012; 27:560-574.

13- 13. Márquez Arabia J, Suárez G, Márquez Tróchez J. Papel del ejercicio en la prevención de la diabetes tipo 2. Rev. cuba. endocrinol. 2010; 21(2)182-201

14- 14. Organización Mundial de la Salud. Estrategia mundial sobre régimen alimentario, actividad física y salud: actividad física. [online] OMS: Ginebra, 2008 [Accessed 2 Oct. 2018]. Available at: http://www. who.int/dietphysicalactivity/pa/es/

15- 15. Cabalé Vilariño M, Meneau X, Núñez M, Miguélez R, Ferrer M, Rodríguez Nande L. Incidencia de las dislipidemias y su relación con la cardiopatía isquémica en la población del Policlínico "Héroes del Moncada“". [Internet] Rev Cubana Med Gen Integr. 2005[Accessed 2 Oct. 2018]; 21 ( 5-6 ): [aprox. 6 p]. Available at: http://scielo.sld.cu/scielo.php?script=sci_arttext\&pid=S0864-21252005000500002

16- 16. Khoury JC, Kleindorfer D, Alwell K, Moomaw CJ, Woo D, Adeoye O, et al. Diabetes mellitus: a risk factor for ischemic stroke in a large biracial population. Stroke. 2013; 44(6):1500-4.

17- 17. Rojas J, Zurru M, Patrucco L, Romano M, Riccio P, Cristiano E. Registro de enfermedad cerebrovascular isquémica. Medicina (B. Aires). 2006; 66( 6 ): 547-551

18- 18. Braga P, Ibarra A, Rega I, Servente L, Benzano D, Ketzoian C, et al. Ataque cerebrovascular: un estudio epidemiológico prospectivo en el Hospital de Clínicas de Montevideo. Rev Med Uruguay 2001; 17: 42-54.

19- 19. Paul SL, Thrift AG, Donnan GA. Smoking as a crucial independent determinant of stroke. Tob Induc Dis. 2004;2(2):67-80. 
20- 20. Cabrera Zamora JL. Factores de riesgo y enfermedad cerebrovascular. Rev Cubana Angiol Cir Vasc. 2014; 15(2):75-88

21- 21. Ridker PM, Hennekens CH, Lindpaintner K, Stampfer MJ, Eisenberg PR, Miletich JP. Mutation in the gene coding for coagulation factor $V$ and the risk of myocardial infarction, stroke, continuous positive airway pressure: and observational study. N Engl J Med. 1995 Apr 6;332(14):912-7.

22- 22. Pebet Arbini M, Soria Vargas VR. Examen neurológico: teoría y técnica síndromes neurológicos descripción y topografía. Montevideo: Bibliomedica,2015. pp.93-209.

23- 23. Taroco R, Fernández A, Vales V, Leizagoyen F, Devoto N. Ataque cerebro vascular (ACV) isquémico. Arch Med Interna. 2009; 31(Supl 1): S34-S45.

24- 24. Higgie J, Urban L, Hackembruch J, Gaye A. Análisis de una Cohorte de Pacientes con ACV del Joven, Hospital de Clínicas, Montevideo. Rev. Urug. Med. Interna. 2018; 2(2): 3-12.

25- 25. García Fernández, M, Bermejo J, Moreno M. Utilidad de las técnicas de imagen en la valoración etiológica y toma de decisiones en el paciente con ictus. Rev Esp Cardiol. 2003;56(11):1119-32

\section{Aporte cadla autor al trabajo}

Javier Gaudiano: 20\%

Diego Graña: $15 \%$

Mabel Goñi: 5\%

Virginia Colina: $10 \%$

Andrea Cosentino: $10 \%$

Romina Pensado: $10 \%$

Victoria Ruglio: 10\%

Magali Scaron: 10\%

Leticia Vidart: 10\% 